\title{
Control of pigeons' matching and mismatching performance by instructional cues
}

\author{
CHARLES A. EDWARDS \\ Dalhousie University, Halifax, Nova Scotia, Canada \\ and \\ JAMES S. MILLER and THOMAS R. ZENTALL \\ University of Kentucky, Lexington, Kentucky
}

\begin{abstract}
Pigeons were trained on a two-stimulus-shape (a plus and a circle) complex conditional discrimination that required birds to match sample and comparison stimuli on some trials and to mismatch on other trials, depending on the level of chamber illumination (bright or dark). Following acquisition, the birds were transferred to a novel color (red and green) task. For half of the birds, the contingencies between levels of illumination and the match/mismatch response requirements were consistent with training (nonreversal condition). For the remaining birds, the contingencies between levels of illumination and match/mismatch response requirements were the opposite of those established in training (reversal condition). Birds in the nonreversal condition acquired the color match/mismatch task at a significantly faster rate than birds in the reversal condition. These results indicate that relation-based responding (generalized matching/ mismatching) is subject to discriminative control.
\end{abstract}

Two tasks that have been used extensively to study "same/different" learning in pigeons are matching-tosample (MTS) and oddity-from-sample (OFS) (Carter \& Werner, 1978; Urcuioli, 1977; Zentall \& Hogan, 1974, 1976). Both tasks require that birds first respond to a conditional or sample stimulus, and then choose between matching and nonmatching comparison stimuli. A peck to the matching comparison is reinforced under the MTS procedure, whereas a peck to the nonmatching comparison is reinforced under the OFS procedure. Because either task can be trained using the same set of sample and comparison stimuli (as well as the same trial and intertrial parameters and sample-response requirements), the difference between the tasks is essentially a difference in the experimenter's designation of the correct choice alternative. Thus, MTS and OFS provide a set of complementary tasks that allow one to make direct comparisons of matching and oddity learning while holding all other factors constant.

The assumption that underlies the use of MTS and OFS tasks as procedures for assessing same/different learning

The authors wish to thank Donald F. McCoy for providing the birds used in the present study and Joyce A. Jagielo for her help in running subjects. Marcia L. Spetch and Werner K. Honig provided many helpful comments on an earlier version of the manuscript. Portions of these data were presented at the meeting of the Midwestern Psychological Association, May 1983. Requests for reprints should be sent to Charles A. Edwards, Department of Psychology, Dalhousie University, Halifax, NS, Canada B3H 4Jl. is that the relation between the sample and the correct alternative ("same" in MTS, and "different" in OFS) can serve as the basis for a generalized "match" or "mismatch" response rule. If birds adopt a generalized strategy when trained on a MTS or OFS task, then one would expect the birds to respond appropriately when given a novel MTS or OFS problem.

Evidence that pigeons trained on MTS or OFS tasks can adopt a "match" or "mismatch" response rule is mixed. Several investigators (Carter \& Werner, 1978; Cumming \& Berryman, 1961, 1965; D'Amato \& Salmon, 1983; Farthing \& Opuda, 1974) have failed to find transfer to novel stimuli following training on MTS or OFS tasks. Carter and Werner (1978) concluded, on the basis of their data, that birds learn MTS and OFS tasks by learning a number of stimulus-specific sample-comparison associations, ones that do not involve learning about the relational cues inherent in the task. According to Carter and Werner, birds fail to transfer to novel stimuli simply because they have not had the opportunity to learn the appropriate sample-comparison associations.

Other investigators (e.g., Urcuioli, 1977; Zentall \& Hogan, 1974) have obtained evidence that same/different cues can have a significant effect on transfer to novel MTS or OFS problems. In an early experiment, Zentall and Hogan (1974) trained one group of pigeons on a red-green MTS task. A separate group of birds were trained on a red-green OFS task. Following acquisition, the birds were tested with a novel blue-yellow MTS or OFS problem. Half of the birds in each of the training groups were main- 
tained on the same task when the blue-yellow problem was introduced (i.e., some birds were trained on red-green MTS and tested with blue-yellow MTS; other birds were trained and tested with OFS). The remaining birds in each training group were shifted to the opposite task when the blue-yellow problem was introduced (i.e., some birds were trained on red-green MTS and tested with blueyellow OFS; others were trained on OFS and tested with MTS).

The transfer results indicated that performance for birds maintained on the same task from training to testing was significantly higher than performance for birds that were shifted to the opposite task when the novel blue-yellow problem was introduced. However, transfer was not allor-none. The overall difference between nonshifted and shifted conditions on the first session of transfer was $13 \%$ (60\% correct responding for birds in nonshifted conditions vs. $47 \%$ correct for birds in shifted conditions). The level of performance obtained on the first session of transfer could be attributed to disruption produced by the novelty of the test stimuli. However, in subsequent sessions, when novelty effects would presumably have dissipated, birds' performance did not shift in a direction consistent with their prior training. Instead, performance in subsequent sessions improved for both groups at comparable rates. This pattern of results indicates that there was considerable stimulus-specific learning during the test phase (and, presumably, during original training). Nonetheless, the results demonstrated that pigeons' behavior during testing was at least partially controlled by the relations between sample and comparison stimuli.

Subsequent replications of Zentall and Hogan's initial finding (Zentall \& Hogan, 1976, 1978) have reliably established that pigeons can use same/different cues when transferred to novel MTS or OFS problems. However, the conditions that may be required to demonstrate relation-based responding are not well defined. Part of the difficulty of specifying these conditions is due to the fact that MTS and OFS tasks do not allow one to gain direct control over a relation-based response strategy. Although MTS and OFS training may provide an opportunity for birds to use the relational cues inherent in the stimulus displays, training birds on either of these tasks does not actually require that birds attend to these cues. In a sense, the relational cues are redundant cues, and thus transfer to a novel MTS or OFS problem can only indicate the extent to which birds will "spontaneously" attend to and use relational cues.

The purpose of the present experiment was to extend previous findings by asking whether relation-based responding was subject to discriminative control. Specifically, we wanted to ask whether pigeons could be in structed (across trials) to match and mismatch samples and comparisons, and whether the match/mismatch instructions would control choice performance in the presence of novel sample and comparison stimuli.

We attempted to bring responding based on same/different relations under discriminative control in the following manner. Birds were initially trained on a shape (a plus and a circle) two-choice conditional discrimination task that required the birds to match sample and comparison stimuli on some trials and to mismatch sample and comparison stimuli on other trials. The level of chamber illumination (bright or dark) was manipulated across trials to provide an instruction to either match or mismatch. Thus, birds could not learn the task by learning associations between each sample stimulus and one of the comparison stimuli, since for each sample stimulus the value of the correct comparison was reversed across bright and dark houselight conditions.

In principle, birds could learn a task of this sort without using the "same" or "different" relations. Specifically, birds might solve the task by learning a number of stimulus-specific, higher order conditional rules (e.g., " if stimulus A is present and 'plus' appears as the sample, choose "plus" "; "if stimulus B is present and 'plus' appears as the sample, choose "circle",'; see Santi, 1978). However, we reasoned that birds might be less likely to use these kinds of stimulus-specific rules if the instructional cues were located away from the response keys, and if the instructional cues were diffuse (rather than localizable). Accordingly, the test-chamber houselight was used to signal the matching/mismatching response contingencies.

The instructions (houselight/no houselight) and the response requirements (match/mismatch) were counterbalanced across two groups of birds during training. To further differentiate the match/mismatch contingencies, correct responses on houselight-cued trials were reinforced with one kind of grain, whereas correct responses on no-houselight-cued trials were reinforced with a different kind of grain (see Peterson, 1983).

Control by houselight/no-houselight instructions was assessed by transferring birds to a novel color (red and green) match $/ \mathrm{mismatch}$ problem. For half of the birds, the instructional cues and the match/mismatch response contingencies were consistent with training (nonreversal condition). For the remaining birds the instructional cues and match/mismatch contingencies were reversed when the red-green problem was introduced (reversal condition). We reasoned that if the levels of illumination gained discriminative control over generalized matching and mismatching behavior, then birds in the nonreversal condition would acquire the color task at a faster rate than would birds in the reversal condition. We also expected that performance on both the matching component and the oddity component of the task would be equally affected by the reversal manipulation. On the other hand, if birds acquire a match/mismatch task by learning stimulus-specific rules, then the instruction-reversal manipulation would not be expected to have any differential affect on acquisition of the color task.

\section{METHOD}

\section{Subjects}

Twelve experimentally naive White Carneaux pigeons, approximately 1 year old at the start of the experiment, were food deprived 
and maintained at $80 \%-85 \%$ of their free-feeding weights throughout the experiment. The birds were individually housed in wire-mesh cages. Free access to water and grit was provided.

\section{Apparatus}

The apparatus was a standard operant chamber that measured $30 \mathrm{~cm}$ wide, $35 \mathrm{~cm}$ high, and $35 \mathrm{~cm}$ across the stimulus panel. The panel contained three horizontally aligned rectangular response keys $(3.2 \times 2.5 \mathrm{~cm})$, separated by $2.0 \mathrm{~cm}$, edge to edge. The bottom edges of the response keys were $16.0 \mathrm{~cm}$ above a wire-mesh floor. IEE in-line projectors, located behind each response key, were used to illuminate the keys with red or green hues (Kodak Wratten filters No. 26 and No. 60 , respectively), or one of two white shapes (plus or circle) on a black background. The plus consisted of a horizontal and a vertical line, each $18 \mathrm{~mm}$ long and $3 \mathrm{~mm}$ wide, that intersected at the midpoints. The circle was a line-drawn annulus with an outside diameter of $16 \mathrm{~mm}$ and an inside diameter of $13 \mathrm{~mm}$. A shielded 28-V dc bulb (GE 1218), centered in the ceiling, served as the houselight. This bulb was connected to a ground source through a simple diode-resistor matrix such that three levels of chamber illumination could be produced: "bright" (no resistance in the bulb circuit); "dim" (a single 122- $\Omega$ resistor in series with the bulb); and "dark" (no current to the bulb).

Two solenoid-operated grain feeders were backmounted to the stimulus panel. Each feeder could be raised to the same $5.8 \times 5.0 \mathrm{~cm}$ reinforcement access opening, the bottom edge of which was $7.0 \mathrm{~cm}$ above the wire-mesh floor and centered below the middle response key. One hopper contained whole wheat (obtained from a local farm supply), and the other hopper contained split peas (Kroger Co.).

BRS solid-state equipment, located in an adjoining room, controlled the presentation of stimulus events and recorded response data. Sound masking was provided by white noise and a blower fan.

\section{Procedure}

Pretraining. The birds were first trained to eat from the food hopper, then autoshaped to peck a white light (CS) presented on the center key. During the five sessions of autoshaping, 8-sec CS presentations were followed by 3 -sec access to randomly ordered presentations of peas or wheat. Pecks during the 8-sec CS interval terminated the white light and were immediately reinforced. Autoshaping trials were separated by a $30-\mathrm{sec}$ intertrial interval (ITI). The dim houselight remained on throughout each autoshaping session.

On Session 6 of pretraining, the ITI was reduced to $5 \mathrm{sec}$, the reinforcement cycle was reduced to $2.5 \mathrm{sec}$, and a single response was required to produce reinforcement. The white light was replaced by plus and circle stimuli, each of which was presented 10 times on each of the three response keys in a random order. The chamber remained dark during the ITI and on a random half of the singlestimulus trials, but the chamber was illuminated with the bright houselight on the remaining single-stimulus trials. The birds were reinforced with either peas or wheat during single-stimulus training. The kind of reinforcer was uncorrelated with plus and circle stimuli, but was correlated with houselight/no-houselight trials; the birds were reinforced with peas when the chamber was bright during a trial, but were reinforced with wheat when the chamber remained dark during a trial. The response requirement was increased to a fixed ratio of 10 responses over the next 7 sessions of singlestimulus training.

Shape training. On the day following the last pretraining session, the birds were placed on a two-stimulus match/mismatch task. A trial was initiated by illuminating the center response key with either a plus or a circle sample. Ten pecks to the sample were required to turn on comparison stimuli, presented on the side keys. A single peck to the correct comparison (defined below) was reinforced with a 2.5 -sec access to food and initiated a 5-sec ITI. A peck to the incorrect comparison was followed only by the ITI. The circle and plus appeared equally often as samples and comparisons within each daily session of 96 trials. Trials were randomized with the following constraints: the same stimulus could not appear as the sample on more than three consecutive trials; the correct comparison could not appear on the same side (left or right) on more than three consecutive trials; and the same response (matching or mismatching) was not scheduled as the correct response on more than three consecutive trials.

Forty-eight matching trials and 48 oddity trials were presented in each session. On half of the trials, the bright houselight came on when the sample was presented and remained on until a choice response was made. On the remaining trials, the houselight remained off throughout the trial. One group of birds, designated houselightmatch (or Group HM, $n=6$ ) was reinforced for matching when the bright houselight was on during a trial but for mismatching when the chamber remained dark during a trial. A second group of birds, designated houselight-odd (or Group $\mathrm{HO}, n=6$ ), was reinforced for mismatching when the bright houselight remained on during a trial but for matching when the chamber remained dark during a trial. Correct responses for birds in both groups were reinforced with peas when the bright houselight was present during a trial and with wheat when the chamber remained dark during a trial. These conditions were in effect for 44 sessions.

Beginning on Session 45 , the chamber was illuminated with a dim light during all intertrial intervals for both groups, and training continued for an additional 40 sessions.

Color testing. On Session 87, a novel color (red and green) match/mismatch problem was introduced (no shape trials were presented during the color-testing phase). Birds in each of the training groups were assigned to either nonreversal or reversal conditions at the beginning of color testing. For birds assigned to the nonreversal condition, the contingencies between the houselight instructions and response requirements were consistent with training. For example, if a bird had learned to match shapes in a bright chamber (and to mismatch shapes in a dark chamber), the bird was required to match colors in a bright chamber (and to mismatch colors in a dark chamber). For birds assigned to the reversal conditions, the contingencies between houselight instructions and response requirements were reversed. For example, a bird trained to match shapes in a bright chamber was required to mismatch colors in a bright chamber.

Forty-eight matching trials and 48 oddity trials were presented during each session of color testing. Constraints on randomization of trial order and trial parameters were the same as those used during shape training. Color testing continued for a total of 16 sessions.

\section{RESULTS}

\section{Shape Training}

Dark intertrial intervals. Two birds in Group HM and one bird in Group HO failed to learn the shape match/mismatch task. Results for these three birds were not included in the analyses of the data and will not be presented.

Acquisition of the shape task is presented in Figure 1. The break in the curves indicates the point at which dim intertrial intervals were introduced.

A two-way mixed design analysis of variance for unequal sample sizes was used to analyze the data, with group (HM vs. $\mathrm{HO}$ ) as the nonrepeated factor and instruction (houselight vs. no houselight) as the repeated factor. Overall, performance for Groups HM and HO did not differ during the first 11 blocks (44 sessions) of training $[F(1,7)<1]$. However, the effect of instruction was significant $[F(1,7)=64.43, \mathrm{p}<.001]$. As shown in Figure 1, in each training group, performance on the task cued by the houselight exceeded performance on the task cued by a dark chamber. Thus, matching performance was sig- 


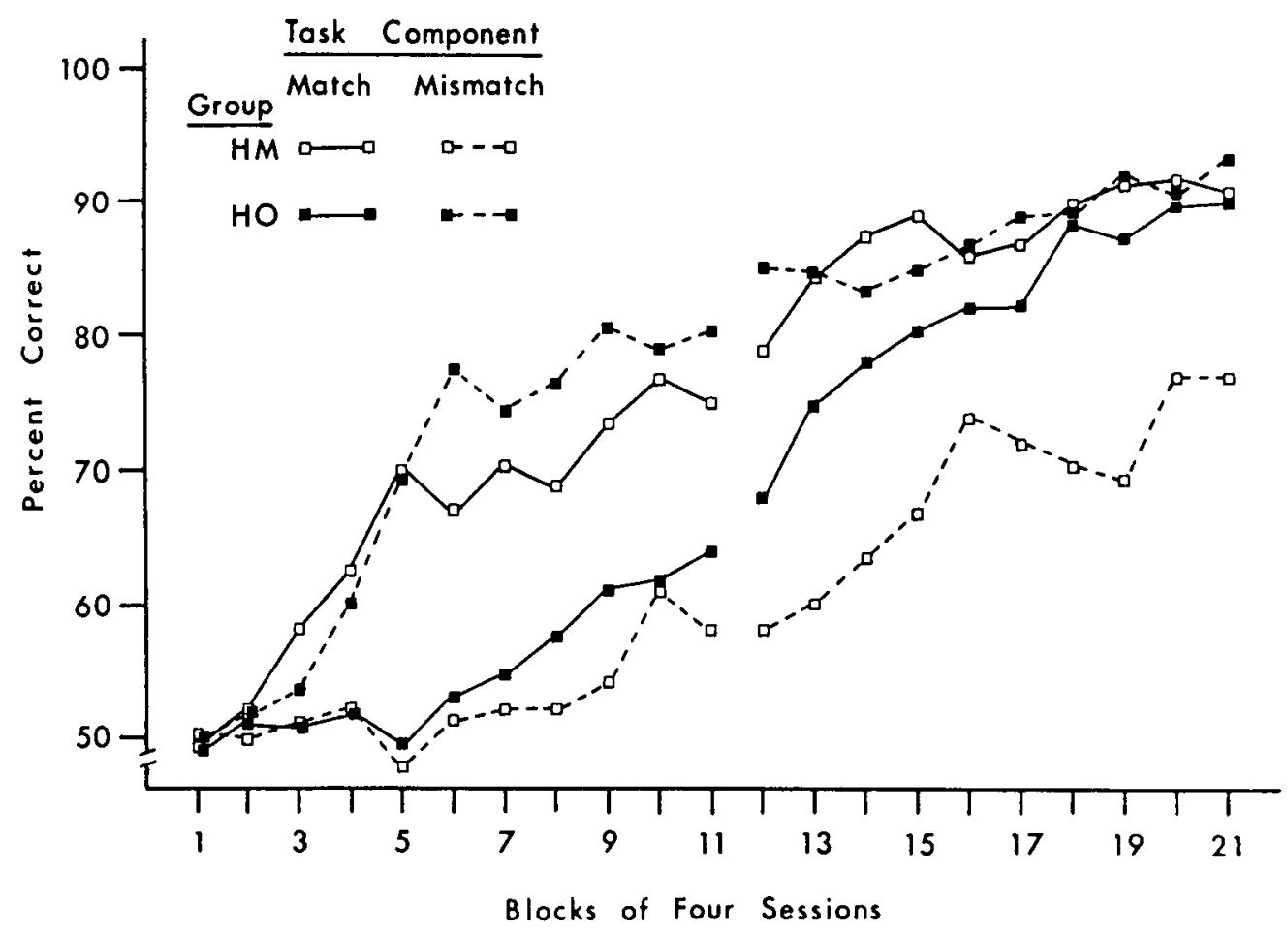

Figure 1. Acquisition of the shape match/mismatch task as a function of training group ("HM" for houselightmatch and "HO" for houselight-odd) and task component (matching and mismatching).

nificantly higher than oddity performance in the houselight-match condition $[65.9 \%$ correct and $52.7 \%$ correct, respectively; $F(1,3)=16.927, p<.05]$, whereas oddity performance was significantly higher than matching performance in the houselight-odd condition $[68.6 \%$ correct and $54.9 \%$ correct, respectively; $F(1,4)=73.858$, $\mathrm{p}<.01]$.

Dim intertrial intervals. The asymmetry in rates of learning the components of the match/mismatch task prompted the change in illumination level during the intertrial. We reasoned that change per se in overall illumination (a change associated with the start of half of the trials) might have contributed to the houselight effect obtained in the first 44 sessions of training. Providing a dim level of illumination during all intertrials insured that a change in illumination would occur at the start of every trial (either dim to dark or dim to bright).

When the data were averaged over the last 10 blocks of training, no difference in the overall level of performance between Group HM and Group HO was obtained $[F(1,7)=2.83, p>.05]$. However, birds continued to perform at a higher level on the tasks cued by the houselight $[F(1,7)=63.05, \mathrm{p}<.001]$ despite the change in ITI illumination. For Group HM, houselight-cued matching performance was significantly higher than dark-cued oddity performance $[87.7 \%$ correct and $68.9 \%$ correct, respectively; $F(1,3)=65.879, p<.01]$. Matching/oddity differences were not as great for Group $\mathrm{HO}$ as they were for Group HM; nonetheless, for Group HO, houselight-cued oddity performance was significantly higher than dark- cued matching performance $[88.2 \%$ correct and $82.2 \%$ correct, respectively; $F(1,4)=8.211, p<.05$ ].

In addition, there was a significant group $x$ instruction interaction $[F(1,7)=16.88, p<.01]$. Post hoc comparisons localized the source of this interaction to a between-groups difference in performance on the nohouselight-cued components of the match/mismatch task. As shown in Figure 1, matching performance for Group $\mathrm{HO}$ was significantly higher than oddity performance for Group HM [82.2\% correct and $68.9 \%$ correct, respectively; $F(1,7)=6.669, p<.05$ ]. However, performance on the houselight-cued component was comparable for Groups $\mathrm{HM}$ and $\mathrm{HO}[\mathrm{F}(1,7)<1]$.

\section{Color Testing}

Acquisition of the color match/mismatch problem is presented in Figure 2. Mean performance for nonreversal and reversal conditions is plotted separately for the matching component (left-hand panel) and the mismatching component (right-hand panel). Each data point represents performance averaged over four sessions. As illustrated in Figure 2, the difference between nonreversal performance (solid lines) and reversal performance (dashed lines) did not appear to be differentially affected by task component.

A two-way mixed design analysis of variance was used to analyze the color-test data, with test condition (nonreversal vs. reversal) as the nonrepeated factor and task component (matching vs. mismatching) as the repeated factor. Only the simple main effect of test condition was 


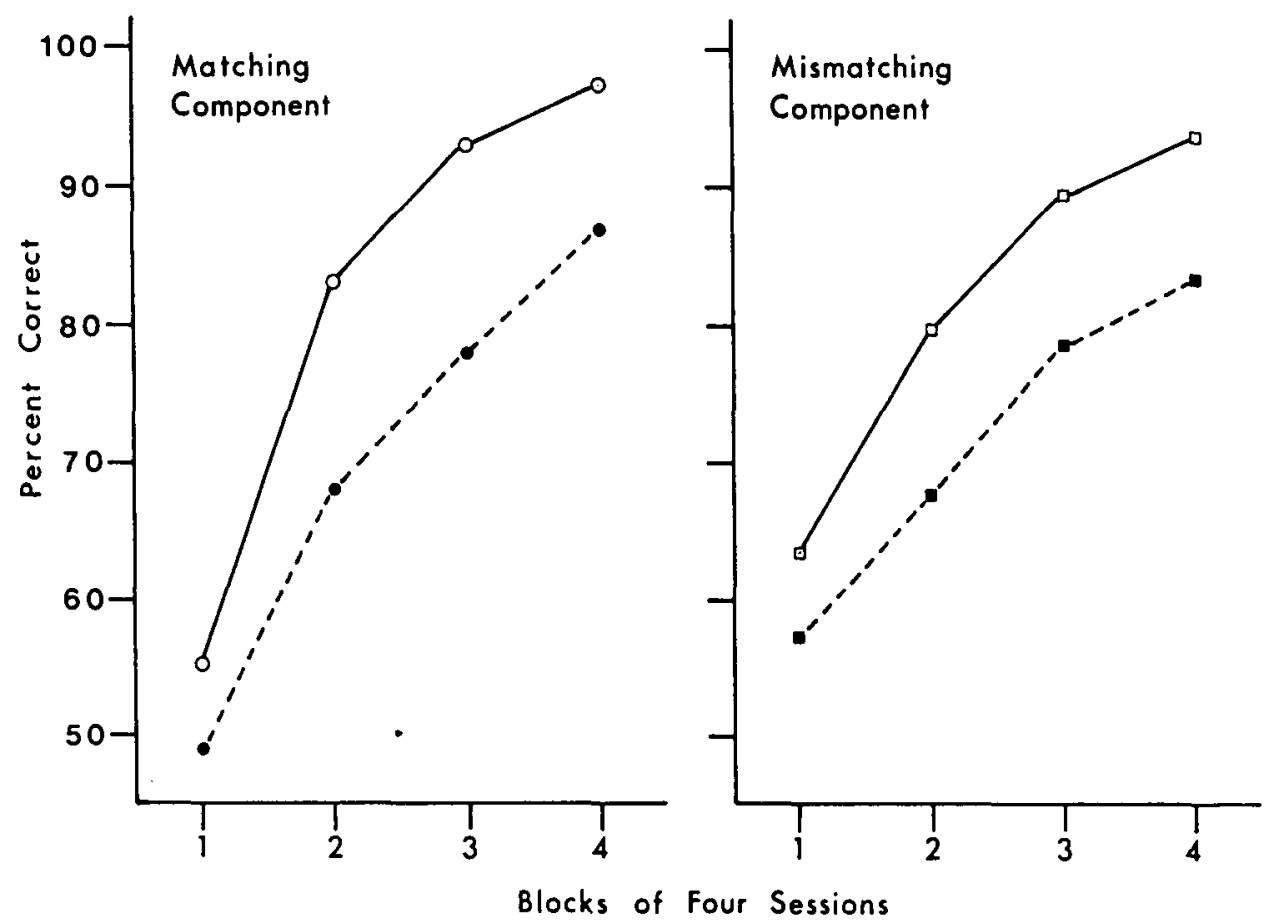

Figure 2. Acquisition of the color match/mismatch task as a function of test condition (nonreversal and reversal) and task component (matching and mismatching). Each data point represents performance for four sessions of testing. Nonreversal performance is represented by the solid lines; reversal performance is represented by the dashed lines.

significant $[F(1,7)=27.77, \mathrm{p}<.01]$. Birds in the nonreversal condition averaged $82.0 \%$ correct over the 16 sessions of testing; birds in the reversal condition averaged $71.2 \%$ correct. Additional comparisons revealed that significant differences in performance were obtained on both the matching component of the task [nonreversal, mean $=82.2 \%$ correct; reversal, mean $=70.5 \%$ correct; $F(1,7)=10.78, p<.025]$ and the oddity component of the task [nonreversal, mean $=81.7 \%$ correct; reversal, mean $=71.8 \%$ correct; $F(1,7)=21.13, p<.01$ ]

Figure 3 shows the course of acquisition for individual birds on each component of the color match/mismatch task. Acquisition for birds originally trained under the houselight-match contingencies is illustrated in the upper half of the figure; acquisition for birds originally trained under the houselight-odd contingencies is shown in the bottom half of the figure. Performance on the matching component of the task appears in the left-hand panels; mismatching performance appears in the right-hand panels.

The clearest difference between nonreversal and reversal performance was obtained on the mismatching component of the task for birds that were originally trained under the houselight-odd contingencies (as shown in the lower right-hand panel of Figure 3). Performance on the matching component of the task (shown in the lower lefthand panel) also shows a large effect of the reversal manipulation, with the exception of the data obtained for Bird 954. The overall difference (i.e., pooled over task components and session blocks) between nonreversal and reversal performance for birds trained under the houselight-odd contingencies was $13.9 \%$ (nonreversal, mean $=85.1 \%$ correct; reversal, mean $=71.2 \%$ correct).

The overall difference between nonreversal and reversal performance was not as great for birds originally trained under the houselight-match contingencies (nonreversal, mean $=78.7 \%$ correct; reversal, mean = $71.4 \%$ correct). When the data were pooled over the two task components, performance for the two birds assigned to the nonreversal condition was consistently higher than performance for the two birds assigned to the reversal condition on each of the four session blocks of testing. The differences were not as consistent, however, when performance was considered separately for each of the task components (as shown in the upper-left and upper-right panels of Figure 3). We suspect that the smaller nonreversal versus reversal difference obtained for birds originally trained under the houselight-match contingencies reflects the lower level of performance achieved at the end of the shape training, specifically the lower level of performance on the no-houselight-cued (mismatching) component of the shape task.

Because the level of chamber illumination differentially controlled acquisition of the match/mismatch components of the task during shape training, it seemed prudent to ask whether there was any evidence that illumination per se differentially affected the pattern of results obtained during the color testing phase. However, an examination of the data indicated that each bird's performance on the houselight-cued-task component was comparable, over- 

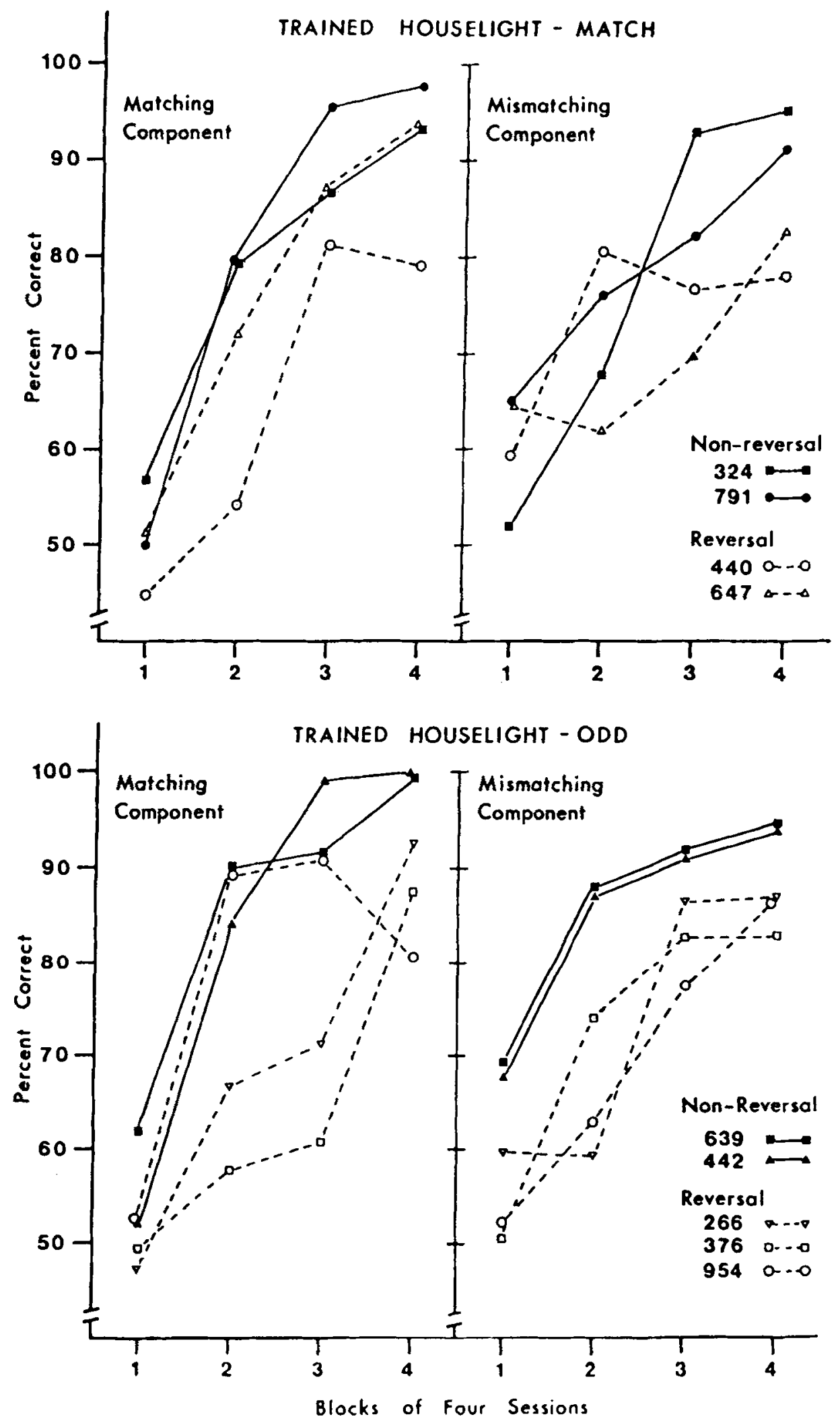

Figure 3. Acquisition of the color match/mismatch task for individual birds. Performance of birds originally trained under the houselight-match contingencies is shown in the upper half of the figure; performance of birds originally trained under the houselight-odd contingencies is shown in the lower half of the figure. Matching performance is shown in the left-hand panels; mismatching, in the righthand panels. 
all, to its performance on the dark-cued-task component. Although there was greater within-subject variability in performance for birds in the reversal conditions, these birds showed no consistent tendency to perform at a higher level on the houselight-cued-task component than on the dark-cued-task component. A post hoc repeated measures analysis of variance that compared houselight-cued and dark-cued performance (pooled over test condition) confirmed this impression $[F(1,8)<1]$.

\section{DISCUSSION}

The contribution of the present experiment to the existing literature was the demonstration that relation-based responding is subject to discriminative control. Pigeons were first trained on a higher order conditional discrimination task, one that required birds to either match or mismatch shape sample and comparison stimuli, depending on the level of chamber illumination (houselight and nohouselight instructions). Evidence that the instructional cues controlled a generalized match/mismatch response strategy was obtained when novel sample and comparison stimuli (colors) were substituted for the shape stimuli. During the color-test phase, birds for which the instructions and match/mismatch response contingencies were consistent with training (nonreversal condition) showed faster acquisition of the color task, relative to performance for birds tested under the reversal condition. The difference between nonreversal and reversal performance indicated that birds did not acquire the color task simply by learning a new set of stimulus-specific choice response rules; rather, the transfer data indicated that the houselight instructions controlled a response strategy that was to some degree independent of the specific sample and comparison stimulus values.

The results obtained in the present experiment are consistent with those previously reported by Zentall and Hogan, in particular their shape-to-color transfer data (Zentall \& Hogan, 1976, 1978). They found that birds maintained on the same task from training to transfer (matching to matching or oddity to oddity) performed at a higher level than birds shifted to the opposite task (matching to oddity or oddity to matching). However, several investigators (Carter \& Werner, 1978; D'Amato, Salmon, \& Columbo, 1985; Mackintosh, 1983) have questioned whether Zentall and Hogan's results actually demonstrated transfer of a relation-based response strategy, or whether the differences between nonshifted and shifted groups might have been due to nonrelational factors. We will first review the major alternative explanation that has been proposed to account for the earlier transfer data, and then consider whether the present results are open to a similar explanation.

Carter and Werner (1978) pointed out that the effect of shifting birds from one task to the other task in Zentall and Hogan's experiments was not always symmetrical. In two studies (Zentall \& Hogan, 1974, 1976), the per- formance of birds trained with oddity and shifted to matching was below nonshifted performance (as expected), but the performance of birds trained with matching and shifted to oddity was comparable to nonshifted performance. Thus, the overall difference between nonshifted and shifted performance appeared to be due to negative transfer for one of the two shifted groups, rather than positive transfer for the two nonshifted groups (Carter \& Werner, 1978; Mackintosh, 1983).

D'Amato et al. (1985) attributed the asymmetry to an oddity bias. D'Amato et al. noted that when birds are first exposed to either a MTS or an OFS task, birds show an initial tendency to respond to the nonmatching comparison, regardless of the task (e.g., Cumming \& Berryman, 1965). This oddity bias can be accounted for by noting that sample responses are never reinforced, so that birds avoid pecking that stimulus when it appears as a choice stimulus. With regard to the asymmetry in transfer performance for shifted groups in Zentall and Hogan's studies, D'Amato et al. argued that birds that were initially trained on MTS would have to learn to inhibit responses to the nonmatching comparison stimulus. However, when shifted to the OFS task, birds could simply revert to what was a "natural" tendency (i.e., respond to the nonmatching comparison). Thus, the performance of birds shifted from matching to oddity was comparable to nonshifted performance because the oddity bias was consistent with the transfer task contingencies. On the other hand, birds that were first trained on OFS did not have to learn to inhibit responses to the nonmatching comparison (indeed, this tendency would be strengthened by OFS training) until they were subsequently shifted to the MTS task. As a consequence, birds that were shifted from oddity to matching made more errors in transfer than nonshifted birds because they had not yet learned to inhibit responses to the nonmatching comparison stimulus (the incorrect alternative in transfer).

Although this explanation may account for some of the earlier data reported by Zentall and Hogan (1974, 1976), it does not account for all of the differences between nonshifted and shifted performance in a more recent study (see Zentall \& Hogan, 1978). More importantly, an oddity bias does not seem to account for the difference between nonreversal and reversal performance obtained in the present experiment. The design of the present study insured that birds had equivalent experience with both matching and mismatching trials prior to transfer, and the results indicated that the difference between nonreversal and reversal performance was comparable on both the matching and mismatching components of the task.

We suggested in the introduction that previous failures to demonstrate transfer of MTS or OFS might be due to the fact that training a bird on either a MTS or an OFS task does not, by itself, guarantee that the bird will actually attend to the relations between sample and comparison stimuli. However, one might still ask why birds do not show greater "spontaneous" use of the relational cues 
inherent in these tasks, and why, on the other hand, instructions to match and mismatch might promote the use of these cues.

Premack (1978) has provided one possible explanation for birds' failure to transfer to novel MTS or OFS problems. According to Premack, MTS and OFS tasks pit the absolute properties of sample and comparison stimuli (e.g., "red," "vertical," etc.) against their relational properties (e.g., "same"). Presumably, the degree to which any species shows transfer from one MTS or OFS problem to a second MTS or OFS problem serves to index an animal's capacity to use the "abstract relations" of same and different. The fact that pigeons show little or no transfer to novel problems suggests to Premack and others (e.g., D'Amato et al., 1985) that birds have only a limited capacity to evaluate these relations.

However, one might make an additional distinction between the physical properties of the sample and comparison stimuli (whether absolute or relational) and the differences in the functional roles of those stimuli. If one wanted to ask whether an animal could judge that one stimulus was physically identical to (or different from) a second stimulus, one would want to ensure that the two stimuli being compared were not otherwise differentiated. This condition is not satisfied in MTS and OFS tasks. Samples and comparisons differ from each other along a number of dimensions, such as their temporal occurrence during a trial, and the differential consequences of responding to each of these stimuli. Furthermore, each sample serves as a unique instruction to direct responding to a particular choice stimulus (Carter \& Werner, 1978). Thus, the sample stimulus is not functionally the same as either of the comparison stimuli in MTS and OFS tasks. Birds might fail to transfer because the structure of both tasks may promote attention to functional relationships ("if sample A, choose comparison A"), rather than to physical relations ("sample $A$ is the same as comparison $A$ ").

The role of the houselight in the present experiment might perhaps be understood in terms of differences in the functional significance of the various cues used to train the task. Specifically, functional differences between samples and comparisons may have been reduced in the match/mismatch task because the houselight acquired a function (that of directing choice behavior) that is otherwise assumed by the sample stimulus in MTS and OFS tasks. Accordingly, birds trained on the match/mismatch task may have assigned less weight to the rules relating specific samples to specific comparisons, and greater weight to the relational cues inherent in the response key stimulus display.

It is also possible that houselight cues produced their effects in transfer because they served as (differential) retrieval cues for the shape training task. The possibility that memory may be important to transfer performance has not been considered in previous discussions of same/different learning. Nonetheless, it would seem that memory for some aspect of the training task is a prerequisite for transfer. A bird might fail to transfer, say, from a shape MTS problem to a color MTS problem because there are no cues contained in the color stimuli to prompt memory of the previous shape task.

If houselight cues did, in fact, serve a memory retrieval function in transfer, one might ask about the content of the memories evoked by the houselight cues. One possibility is that birds attended to the relational cues in training and associated a response rule with those cues (e.g., "match sample and comparison stimuli when the houselight is on"). The houselight cues would then serve to retrieve the appropriate rule in transfer.

A second possibility is that the houselight cues may have retrieved memory for specific associations ("if houselight and 'plus' sample, choose 'plus'"), ones that were not evaluated in terms of their relational content until the novel color problem was introduced. In other words, an animal may fail to recognize that a relational cue is (potentially) a relevant cue for choice behavior until that same cue is instantiated by a novel set of stimuli. What is implied here is that birds do not "learn" to use a relational cue on the basis of one instance or exemplar of the cue (e.g., Premack, 1978); rather, birds learn to use relational cues through a process of comparing their memory for the earlier task with the content of the current task. The notion that birds might not make the relational comparisons until transferred to a novel problem may account for why there was no initial (i.e., first session) difference between nonreversal and reversal performance in the present experiment, and why on the other hand, differences emerged across sessions of testing.

The emphasis here is not so much on what a bird learns during training ("if... then..." rules vs. concepts of "same and different"), but rather on what a bird remembers during transfer and how it processes its memory for previous experience. We should keep in mind that the transfer designs typically used to study same/different learning do not allow animals to make direct comparisons between the relational content of one set of stimuli (shapes) and the relational content of a second set of stimuli (colors). This comparison process must be mediated by memory mechanisms, and thus retrieval becomes an important factor that may determine whether birds (or any species) show transfer from one problem to another.

Our discussion of the present results has focused on the role of attention and memory in transfer of generalized matching/mismatching response strategies. As such, it departs from a tradition in the literature on same/different learning of attributing positive findings to conceptual capacity (e.g., Zentall \& Hogan, 1974) and negative findings to a lack of capacity (e.g., D'Amato et al., 1985). We may not gain much in the way of an understanding of the phenomenon of relation-based responding by saying that an animal transfers from one matching problem to a second matching problem because it has acquired a "matching concept." And we can never be sure that failures to transfer represent the absence of a capacity to evaluate relational cues, since the failures may indicate nothing more than the lack of experimental control. 


\section{REFERENCES}

Carter, D. E., \& Werner, T. J. (1978). Complex learning and information processing by pigeons: A critical analysis. Journal of the Experimental Analysis of Behavior, 29, 565-601.

Cumming, W. W., \& Berryman, R. (1961). Some data on matching behavior in the pigeon. Journal of the Experimental Analysis of Behavior, 4, 281-284.

Cumming, W. W., \& Berryman, R. (1965). The complex discriminated operant: Studies of matching-to-sample and related problems. In D. I. Mostofsky (Ed.), Stimulus generalization. Stanford, CA: Stanford University Press.

D'Amato, M. R., \& Salmon, D. P. (1983). Cognitive processes in cebus monkeys. In H. L. Roitblat, T. G. Bever, \& H. S. Terrace (Eds.), Animal cognition. Hillsdale, NJ: Erlbaum.

D'Amato, M. R., Salmon, D. P., \& Columbo, M. (1985). Extent and limits of the matching concept in monkeys (Cebus apella). Journal of Experimental Psychology: Animal Behavior Processes, 11, 35-51.

Farthing, G. W., \& Opuda, M. J. (1974). Transfer of matching-tosample in pigeons. Journal of the Experimental Analysis of Behavior, 21, 199-213

MaCkintosh, N. J. (1983). Conditioning and associative learning. New York: Oxford University Press.
Peterson, G. B. (1983). How expectancies guide behavior. In H. L. Roitblat, T. G. Bever, \& H. S. Terrace (Eds.), Animal cognition. Hillsdale, NJ: Erlbaum.

Premack, D. (1978). On the abstractness of human concepts: Why it would be difficult to talk to a pigeon. In S. Hulse, H. Fowler, \& W. Honig (Eds.), Cognitive processes in animal behavior. Hillsdale, $\mathrm{NJ}$ : Erlbaum.

SANTI, A. (1978). The role of physical identity of the sample and correct comparison stimulus in matching-to-sample paradigms. Journal of the Experimental Analysis of Behavior, 29, 511-516.

URCUIOLI, P. (1977). Transfer of oddity-from-sample performance in pigeons. Unpublished master's thesis, Dalhousie University.

Zentall, T. R., \& Hogan, D. E. (1974). Abstract concept learning in the pigeon. Journal of Experimental Psychology, 102, 393-398.

Zentall, T. R., \& Hogan, D. E. (1976). Pigeons can learn identity, or difference, or both. Science, 191, 408-409.

ZENTALl, T. R., \& HogaN, D. E. (1978). Same/different concept learning in the pigeon: The effect of negative instances and prior adaptation to the transfer stimuli. Journal of the Experimental Analysis of Behavior, 30, 177-186.

(Manuscript received March 25, 1985; revision accepted for publication September 23, 1985.) 